\title{
Étude de la stabilité in vitro de 21 antidépresseurs et de 16 neuroleptiques dans le sang total
}

\section{Study of the in vitro stability of 21 antidepressants and 16 neuroleptics in whole blood}

\section{Gaëlle DUFFORT $^{(1)}$, Hélène EYSSERIC ${ }^{(2)}$, Marc DEVEAUX ${ }^{(1)}$, \\ Véronique DUMESTRE-TOULET ${ }^{(3)}$, Marie-Hélène GHYSEL ${ }^{(4)}$,} Jean-Pierre GOULLÉ( ${ }^{(5)}$, Alain GRUSON(ต), Pascal KINTZ ${ }^{(7)}$, Gérard LACHÂTRE ${ }^{(8)}$, Michel LHERMITTE ${ }^{(9)}$, Patrick MURA ${ }^{(10)}$, Anne-Laure PELISSIER-ALICOT ${ }^{(11)}$, Martine PERRIN $^{(12)}$, Ivan RICORDEL ${ }^{(13)}$, Alain TURCANT ${ }^{(14)}$, Gilbert PÉPIN ${ }^{(1) *}$ (Groupe de travail “toxicologie judiciaire" de la Société Française de Toxicologie Analytique)

(1) Laboratoire Toxlab, Paris

(2) Laboratoire de Médecine Légale, UFR de Médecine, Grenoble

(3) Laboratoire Toxgen, Bordeaux

(4) Laboratoire de Police Scientifique, Section Toxicologie, Lille

(5) Laboratoire de Toxicologie, GH, Le Havre

(6) Laboratoire de Biochimie, $\mathrm{CH}$, Arras

(7) Laboratoire Chem Tox, Illkirch

(8) Service de Pharmacologie et Toxicologie, CHRU, Limoges

(9) Laboratoire de Biochimie, CHU, Lille

(10) Laboratoire de Biochimie et de Toxicologie, CHU, Poitiers

(11) Service de Médecine Légale, Faculté de Médecine, Marseille

(12) Département de Toxicologie IRCGN, Rosny sous Bois

(13) Laboratoire de Toxicologie de la Préfecture de Police, Paris

(14) Service de Pharmacologie et Toxicologie, CHU, Angers

*Auteur à qui adresser la correspondance : Gilbert PÉPIN, Laboratoire Toxlab, 7, rue Jacques Cartier 75018 PARIS - FRANCE - Tél : +33 158592800 - Fax : +331585928 01 -E-mail : toxlab@wanadoo.fr

Ce travail a été présenté, partiellement, au $12^{\text {eme }}$ congrès annuel de la SFTA, Porticcio, 4-7 mai 2004 


\section{RÉSUMÉ}

L'interprétation des concentrations sanguines postmortem des xénobiotiques est une étape essentielle de l'expertise toxicologique. La stabilité in vitro des molécules est un paramètre à prendre en compte dans l'interprétation des résultats. Devant le manque de données concernant une éventuelle dégradation in vitro des antidépresseurs et neuroleptiques, le groupe de travail "toxicologie médico-légale » de la Société Française de Toxicologie Analytique (SFTA) a réalisé une étude à partir de cas réels, suivie d'une étude multicentrique sur sangs surchargés. Les résultats obtenus sont très disparates d'une molécule à l'autre, et il apparaît très difficile d'établir une loi générale sur la stabilité in vitro des antidépresseurs et neuroleptiques dans le sang. Ces résultats permettent en revanche de souligner qu'il est primordial, dans le domaine de la toxicologie postmortem, de prendre en compte l'effet matrice à toutes les étapes de l'analyse, d'œuvrer pour obtenir un ajout systématique d'adjuvant de conservation à concentration efficace dès la réalisation des prélèvements autopsiques, et de conserver si possible les échantillons à $-20^{\circ} \mathrm{C}$ dans les phases pré- et post-analytiques.

\section{MOTS-CLÉS}

Antidépresseurs, neuroleptiques, dégradation, stabilité, sang, postmortem, conservateur, température, toxicologie médico-légale.

\section{Introduction}

La recherche des causes toxiques de mort est l'une des missions prépondérantes en toxicologie médico-légale. L'interprétation des concentrations sanguines postmortem est une étape essentielle et souvent délicate de l'expertise. Outre les phénomènes de redistribution se produisant dans l'organisme après le décès et susceptibles de modifier les concentrations des différentes molécules (1), il faut également tenir compte d'éventuelles variations de concentration dans le sang in vitro (2-4).

Très peu de données scientifiques sont disponibles sur la stabilité in vitro des antidépresseurs et des neuroleptiques, les molécules les plus étudiées étant les produits stupéfiants (5-10) et les benzodiazépines (11-13). Concernant les antidépresseurs, une étude démontre la stabilité de la fluoxétine à $-20^{\circ} \mathrm{C}$ dans le sang mais présente des résultats divergents sur sa stabilité à $+4^{\circ} \mathrm{C}$ (14). Concernant les neuroleptiques, Elliot (15) a comparé la stabilité de l'acépromazine in vitro dans le sang total frais, le sang total post-mortem et le plasma et décrit un phénomène de métabolisation in vitro qu'il attribue à une activité réductase des hématies présentes dans l'échantillon. D'autres articles évoquent le problème de la stabilité in vitro de la clozapine (16), de l'olanzapine $(17)$, de la chlorpromazine $(4,18)$ et de dérivés imipraminiques tels que l'amitriptyline, l'imi-

\section{SUMMARY}

The interpretation of drugs levels in postmortem blood is a main step in forensic toxicology. Since days or weeks may pass between sampling and drug analysis, the drug stability must be established for proper interpretation of results. Considering the lack of data about degradation in vitro of antidepressant and neuroleptics, the Forensic Toxicology Committee of the French Society of Analytical Toxicology (SFTA) carried out a study from real cases followed by a multicentric study with supplemented blood. The results are badly matched, so it seems difficult to establish a general rule conceming the stability in vitro of the antidepressants and neuroleptics. On the other hand, these results emphasize the necessity to take into account the matrix effect, to add a preservative with an effective concentration and to store the samples at $-20^{\circ} \mathrm{C}$ until analyzed.

\section{KEY-WORDS}

Antidepressants, neuroleptics, stability, blood, postmortem, preservative, temperature, forensic toxicology.

pramine et la doxépine (18). Une autre équipe s'est intéressée à la stabilité des antidépresseurs tricycliques ajoutés dans des solutions aqueuses formolées (19). Plus récemment, Holmgren et coll. (20) ont réalisé une étude de stabilité à partir de cas réels portant sur 46 molécules de classes chimiques variées, parmi lesquelles figuraient 12 médicaments antidépresseurs ou neuroleptiques.

D'un point de vue méthodologique, les études de stabilité peuvent être classées en deux groupes. Certaines sont basées sur des échantillons biologiques provenant de cas réels $(7,20)$ et d'autres sur des prélèvements biologiques témoins « surchargés » à des concentrations connues des substances dont on souhaite étudier la stabilité $(6,11,15)$. Dans tous les cas, les prélèvements sont aliquotés, conservés à température variable et de nouveau dosés après différents délais de stockage.

Parmi les nombreux facteurs susceptibles de modifier la stabilité des molécules dans le sang in vitro, figurent principalement le type de contenant, l'ajout d'adjuvant de conservation, la température et la durée de stockage. Le type de contenant (plastique ou verre) peut avoir une influence sur des phénomènes d'évaporation $(4,21)$ ou d'adsorption des molécules. La trimipramine et ses métabolites déméthylés et hydroxylés peuvent ainsi s'adsorber sur le verre (22). L'ajout d'un adjuvant de conservation tel que le fluorure de sodium (NaF) a 
montré son intérêt dans la stabilisation de nombreuses molécules telles que l'éthanol, la cocaïne, les cyanures ou les benzodiazépines. L'EDTA est recommandé pour la conservation sanguine du GHB (23). L'ajout d'acide ascorbique pourrait réduire la dégradation de l'olanzapine (17) ; cependant, l'utilisation d'un anti-oxydant semble avoir un effet inverse sur d'autres molécules comme la chlorpromazine dont les métabolites $\mathrm{N}$ oxyde pourraient être réduits in vitro, et entraîner une augmentation de la concentration du produit parent (4).

Enfin, la température de stockage et la durée de conservation sont des paramètres essentiels à prendre en compte dans la stabilité des molécules in vitro. En effet, le délai séparant le prélèvement autopsique de l'analyse peut-être très long pour de multiples raisons (mise au point ou modifications de la technique de dosage, demandes tardives de contre-expertises, etc...). Il est donc impératif d'étudier la stabilité des molécules in vitro sur de longues périodes.

Malgré toutes les précautions prises, l'altération des substances est parfois inévitable. Un résultat négatif n'indique pas toujours que la molécule recherchée n'était pas impliquée au moment du décès. C'est pourquoi la connaissance des mécanismes de dégradation est également tout à fait essentielle dans le domaine de la toxicologie post-mortem.

Le groupe de travail « toxicologie médico-légale » de la SFTA a donc retenu comme sujet d'étude le problème de la stabilité in vitro des antidépresseurs et neuroleptiques dans le sang total. Une étude préliminaire sur cas réels ayant comme objectif de distinguer les molécules « stables au moins 6 mois », des molécules « instables dès le $1^{\text {er }}$ mois» a été entreprise. Cette étude, d'une durée d'un an (décembre 2001 à décembre 2002), a consisté à sélectionner des expertises achevées pour lesquelles il restait suffisamment d'échantillons sanguins permettant des dosages de contrôle $a$ posteriori. Les effets de l'ajout d'un conservateur et de la température de conservation ont été testés sur ces cas. Parmi les 14 laboratoires représentés dans le groupe de travail, 6 ont pu regrouper leurs résultats concernant 42 cas d'antidépresseurs et 31 cas de neuroleptiques. Ces résultats ont été difficiles à exploiter et n'ont pas permis de répondre à l'objectif initial. Citons l'exemple de 3 cas réels d'autopsie impliquant la fluoxétine et pour lesquels un dosage de contrôle avait été réalisé après 1 mois de conservation à $+4^{\circ} \mathrm{C}$ ou $-20^{\circ} \mathrm{C}$ sur un aliquot de sang fluoré. Les concentrations initiales respectives des 3 cas étaient de 144,408 et $1390 \mu \mathrm{g} / \mathrm{L}$ et les pourcentages de la concentration initiale restant de 80,56 et $35 \%$ à $+4^{\circ} \mathrm{C}$ et de 0,78 et $50 \%$ à $-20^{\circ} \mathrm{C}$. Ainsi, aucune conclusion n'avait pu être formulée quant à la stabilité ou non de la fluoxétine après 1 mois de conserva- tion. Cependant, cette étude préliminaire avait eu le mérite de révéler des disparités directement liées aux cas réels, telles que l'état de putréfaction variable des échantillons et l'échelle des concentrations (les concentrations sanguines d'un même antidépresseur variant selon les cas d'une concentration thérapeutique à une concentration létale). Ces biais méthodologiques ont donc amené la commission à mettre au point une étude multicentrique, à partir d'échantillons surchargés, que nous présentons dans cet article.

\section{Matériel et méthode}

L'étude a été réalisée de décembre 2002 à décembre 2003 au sein de 13 laboratoires réalisant des expertises médico-légales. Les 21 antidépresseurs et les 16 neuroleptiques disponibles sur le marché français (24) ont été répartis entre les différents laboratoires, chaque centre étudiant la stabilité de 1 à 3 molécules. Aucune molécule n'a été étudiée par plusieurs centres à la fois. Le protocole adopté préconisait l'utilisation de sang total humain post-mortem (ou éventuellement de sang provenant d'un établissement de transfusion sanguine et conservé plusieurs jours avant son utilisation), additionné ou non de $\mathrm{NaF}$ à $1 \%$ et surchargé en antidépresseur ou neuroleptique à des concentrations fixées à 0,5 ou $2 \mathrm{mg} / \mathrm{L}$ selon les caractéristiques toxico-dynamiques des molécules (environ 5 fois la concentration thérapeutique). Les surcharges réalisées avec des solutions dans le méthanol ou l'acétonitrile ne devaient pas excéder $5 \%$ du volume final de l'échantillon. Un premier dosage après un temps d'homogénéisation de 2 heures était réalisé pour contrôler la concentration initiale (T0). Ces sangs surchargés étaient ensuite conservés dans des tubes en verre à différentes températures : ambiante, $+4^{\circ} \mathrm{C}$ et $-20^{\circ} \mathrm{C}$. Après des délais fixés à 7,15 et 30 jours pour les prélèvements conservés à température ambiante, et des délais fixés à 1,3 et 6 mois pour les prélèvements conservés à $+4^{\circ} \mathrm{C}$ et $-20^{\circ} \mathrm{C}$, des dosages de contrôle étaient effectués. Chaque laboratoire utilisait sa propre technique de dosage validée. Les préparations d'échantillons ont toujours été basées sur une extraction liquide-liquide à $\mathrm{pH}$ alcalin avec des mélanges de solvants variables selon les laboratoires. Les séparations ont fait appel à la chromatographie en phase gazeuse, couplée à la spectrométrie de masse (CPG/SM, 1 laboratoire), à une détection thermoionique (CPG/NPD, 1 laboratoire) ou à la chromatographie en phase liquide, couplée, à une détection par barrette de diodes (CLHP/BD, 10 laboratoires), à un spectromètre de masse (CHLP/SM, 1 laboratoire), ou à un spectromètre de masse tandem (CHLP/SM/SM, 1 laboratoire). 


\section{Résultats}

Les résultats sont présentés dans les tableaux I (conservation avec $\mathrm{NaF}$ ) et II (sans conservateur). Ils indiquent le pourcentage restant de chaque molécule aux différents temps et selon la température de stockage (la concentration initiale étant de $100 \%$ ). Les molécules ont été classées selon les familles chimiques proposées par Bismuth et coll. (25-27).

Nous avons défini deux catégories de molécules : stables (pour lesquelles il reste encore au moins $60 \%$ de la concentration initiale au bout de 6 mois) et instables (pour lesquelles il reste moins de $60 \%$ de la concentration initiale au bout de 6 mois).

\section{Les neuroleptiques}

\section{Benzamides substitués}

Les molécules de ce groupe (amisulpride, sulpiride, sultopride, tiapride) ont un comportement hétérogène : $100 \%$ de la concentration initiale en sulpiride, conservé 6 mois avec NaF, quelle que soit la température, sont retrouvées et $81,5 \%$ de la concentration initiale en sultopride sont retrouvées après 6 mois de conservation à $-20^{\circ} \mathrm{C}$ avec ou sans $\mathrm{NaF}$, alors que les concentrations en amisulpride présentent des variations importantes quelles que soient les conditions de température et les durées de stockage.

\section{Butyrophénones}

Une relative instabilité a été mise en évidence pour l'halopéridol et le dropéridol. La concentration d'halopéridol a nettement diminué entre le $15^{\text {eme }}$ et le $30^{\text {rme }}$ jour, puis s'est maintenue les 5 mois suivants autour de $50 \%$ de la concentration initiale, avec ou sans conservateur. Entre le $3^{\text {ime }}$ et le $6^{\text {eme }}$ mois, la concentration de dropéridol a augmenté (de $47 \%$ avec $\mathrm{NaF}$ et de $15 \%$ sans $\mathrm{NaF}$ ) dans les échantillons conservés à $-20^{\circ} \mathrm{C}$.

\section{Dibenzodiazépines et dibenzoxazépines}

La clozapine et la loxapine se sont avérées très stables après un stockage de 6 mois, quelle que soit la température, avec ou sans conservateur. En effet, 95 à $99 \%$ de la concentration initiale a été retrouvée dans les échantillons sanguins surchargés.

Contrairement à la clozapine et la loxapine, l'olanzapine s'est révélée très instable à température ambiante, puisque après 1 mois de conservation persistait seulement $5 \%$ de la concentration initiale sans $\mathrm{NaF}$ et 0,5 $\%$ avec $\mathrm{NaF}$.

\section{Phénothiazines}

$\mathrm{Au}$ sein de ce groupe de molécules au noyau comptant trois cycles aromatiques, on distingue trois catégories :

- molécules stables : la cyamémazine est très stable pendant 6 mois lorsqu'elle est conservée avec $\mathrm{NaF}$, et stable sans conservateur (sa concentration augmente même parfois). La prométhazine est stable avec conservateur pendant 6 mois (plus stable à $-20^{\circ} \mathrm{C}$ qu'à $+4^{\circ} \mathrm{C}$ ) et instable sans conservateur (seule 27 à $39 \%$ de la concentration initiale est présente dans l'échantillon après 6 mois de stockage).

- molécules instables : l'alimémazine et la lévomépromazine présentent une élévation de la concentration au $3^{\text {em }}$ mois, puis une diminution. La chlorpromazine (mieux conservée à $-20^{\circ} \mathrm{C}$ qu'à $+4^{\circ} \mathrm{C}$ ), et la thioridazine (dont il ne reste que 16 à $33,5 \%$ de la concentration initiale) sont également instables après 6 mois de stockage, avec ou sans conservateur.

- moléculle très instable : la fluphénazine est très instable (au bout de 3 mois le pourcentage restant n'est plus que de 4 à $20 \%$ de la concentration initiale, selon la température de conservation).

\section{Les antidépresseurs}

\section{Imipraminiques}

9 molécules ont été étudiées. Elles peuvent être classées en trois catégories selon leur stabilité :

- molécules très stables : c'est le cas de la doxépine, la maprotiline et la clomipramine (au minimum $72 \%$ de la concentration initiale est encore présente au bout de 6 mois, quelles que soient les conditions de stockage).

- molécules instables : l'amitriptyline, la trimipramine et l'imipramine sont instables avec ou sans $\mathrm{NaF}$. Elles voient leurs concentrations augmenter considérablement jusqu'au $3^{\text {emc }}$ mois puis diminuer à nouveau.

- molécules très instables : l'amoxapine, la dosulépine et la désipramine sont très instables (les pourcentages des concentrations initiales après 6 mois ne sont pas supérieurs à $19 \%$ quelles que soient les conditions de conservation).

\section{Inhibiteurs de la monoamine oxydase (IMAO)}

Le moclobémide, est relativement stable pendant 3 mois avec ou sans ajout de $\mathrm{NaF}$, mais sa concentration diminue jusqu' au $6^{\text {eme }}$ mois pour atteindre des valeurs comprises entre 37 et $52 \%$ de la concentration initiale.

\section{Antidépresseurs non imipraminiques, non IMAO}

11 molécules de ce type ont été étudiées. Celles-ci ont été réparties en 2 catégories de molécules :

- molécules stables : le milnacipran et la viloxazine sont très stables, avec ou sans $\mathrm{NaF}$, après 6 mois de stockage. La sertraline est très stable pendant 6 mois, avec $\mathrm{NaF}$, à +4 et $-20^{\circ} \mathrm{C}$, très stable sans conservateur à $-20^{\circ} \mathrm{C}$, mais instable sans $\mathrm{NaF}$ à température ambiante et $+4^{\circ} \mathrm{C}$. Le citalopram (mieux conservé avec $\mathrm{NaF}$ ), 
la fluoxétine, la venlafaxine et la mirtazapine sont stables après 6 mois avec ou sans conservateur.

- molécules instables : la miansérine, la tianeptine (très stable pendant 1 mois puis sa concentration diminue de moitié jusqu' au $6^{\text {eme }}$ mois) et la paroxétine (assez stable avec $\mathrm{NaF}$ ) sont instables après 6 mois de stockage.
La fluvoxamine est très instable : après 6 mois de stockage avec ou sans $\mathrm{NaF}$, il ne reste que 7 à $14 \%$ de la concentration initiale dans le flacon de sang; de plus, la concentration en fluvoxamine augmente rapidement pendant le $1^{\text {tr }}$ mois de conservation puis diminue fortement jusqu'au $6^{\text {eme }}$ mois.

Tableau I : Pourcentage de la concentration initiale restant au cours du temps (conservation avec fluorure de sodium).

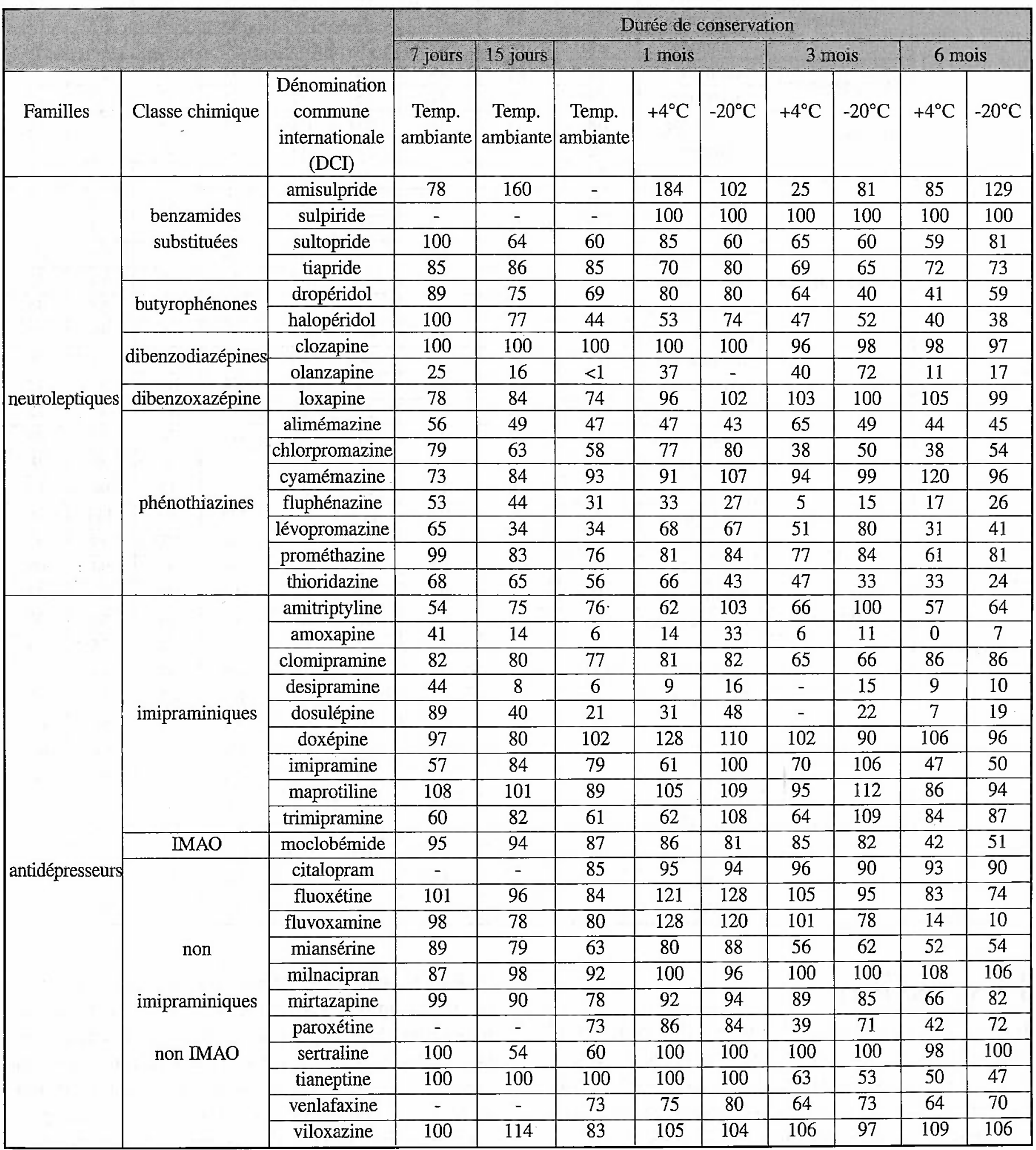


Tableau II : Pourcentage de la concentration initiale restant au cours du temps (sans conservateur).

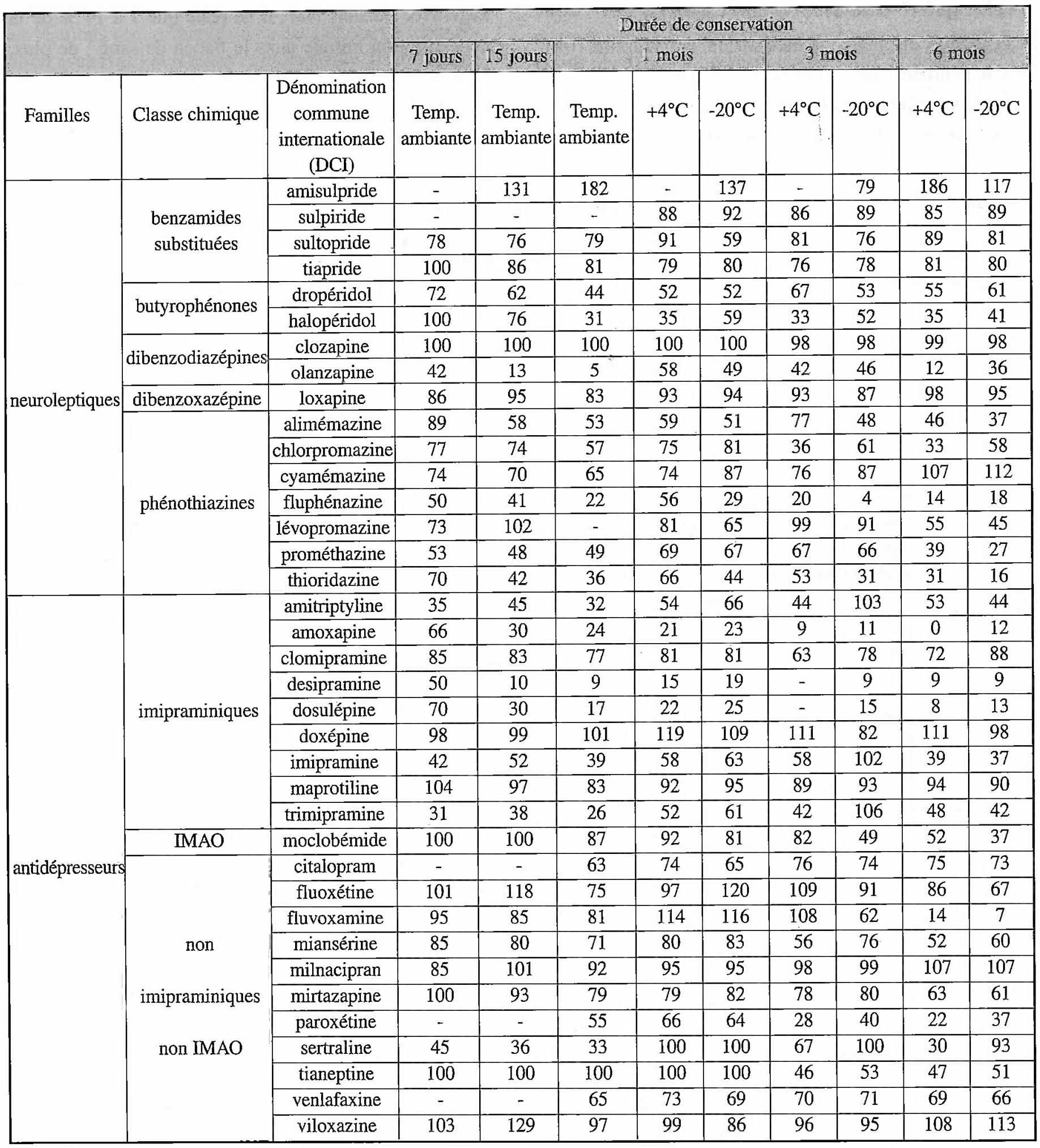

\section{Discussion}

Il existe une grande variabilité de stabilité pour ces 37 molécules, selon leur classe chimique et même au sein d'une classe. Il est donc difficile d'établir une loi générale sur la stabilité d'une famille chimique de neuroleptiques ou d'antidépresseurs. Nous avons recensé 15 molécules stables (le sulpiride, le sultopride, le tiapride, la clozapine, la loxapine, la cyamémazine, la clomipramine, la doxépine, la maprotiline, le citalopram, la fluoxétine, le milnacipran, la mirtazapine, la venlafaxine, la viloxazine), 22 molécules instables (l'amisulpride, le dropéridol, l'halopéridol, l'olanzapine, l'alimémazine, la chlorpromazine, la fluphénazine, la 
lévopromazine, la prométhazine, la thioridazine, l'amitriptyline, l'amoxapine, la désipramine, la dosulépine, l'imipramine, la trimipramine, le moclobémide, la fluvoxamine, la miansérine, la paroxétine, la sertraline, la tianeptine). Parmi ces 22 molécules, 5 d'entre elles présentaient une apparente augmentation de leur concentration initiale (amisulpride, amitriptyline, imipramine, trimipramine et fluvoxamine).

L'ajout de NaF n'a pas permis de mettre en évidence d'amélioration notable de la stabilité des molécules étudiées. En effet, pour la moitié des molécules, aucune différence importante de stabilité n'a été mise en évidence avec $\mathrm{NaF}$; dans $1 / 3$ des cas, la stabilité était meilleure avec $\mathrm{NaF}$ et dans 1 cas sur 6 la stabilité était meilleure sans le NaF.

L'étude en fonction de la température de conservation $\left(+4^{\circ} \mathrm{C}\right.$ et $\left.-20^{\circ} \mathrm{C}\right)$ a montré que la stabilité des molécules était meilleure à $+4^{\circ} \mathrm{C}(1$ cas sur 4$)$ et à $-20^{\circ} \mathrm{C}$ ( 4 cas sur 10). Aucune différence notable n'était observée pour 4 molécules sur 10 .

Malgré tout le soin apporté à l'élaboration de cette étude, en particulier en tenant compte des observations émanant de l'étude préliminaire réalisée à partir de cas réels, la grande hétérogénéité des résultats obtenus pose le problème primordial des biais méthodologiques non maîtrisés.

Le premier biais concerne la disparité de l'origine du sang témoin ayant servi à réaliser les surcharges. Parmi les laboratoires participants, certains n'ont pas pu obtenir du sang post-mortem non putréfié en quantité suffisante, comme cela était préconisé, et ont été contraints de travailler sur du sang provenant de sujets vivants. Deux conséquences importantes en découlent : le statut bactériologique ainsi que les activités enzymatiques plasmatique (estérases) et érythrocytaire (réductases...) des sangs surchargés étaient probablement très différents d'un centre à l'autre. Considérant le phénomène de colonisation par des micro-organismes survenant quelques heures après le décès, ce biais est primordial dans l'étude de la stabilité des substances in vitro, puisque la dégradation enzymatique d'origine bactérienne est un des mécanismes responsables de l'instabilité $(4,28)$. Ce processus peut également expliquer les résultats discordants parfois observés pour une même molécule mais provenant de cas réels différents. Comme cela a déjà été démontré pour la production in vitro d'éthanol (2) ou de cyanures (29), ou inversement pour la dégradation des nitro-benzodiazépines (13) ou du dérivé glucuronide de la morphine (8), le statut bactériologique initial de l'échantillon sanguin, c'est-àdire au moment du prélèvement, est également à prendre en compte pour la stabilité d'autres substances toxiques. Parallèlement, il convient de considérer des phénomènes de dégradation enzymatique tels que l'activité réductase érythrocytaire qui est directement liée à « l'état de fraîcheur » des globules rouges présents (15). L'origine des sangs testés est donc doublement importante lorsque l'on étudie les phénomènes de dégradation in vitro.

En corollaire à ce premier biais, le second concerne l'importance de l'ajout d'un conservateur en quantité efficace. Là aussi, selon les laboratoires participants, la concentration en fluorure a été variable. Soit le conservateur était déjà présent dans les flacons prêts à l'emploi du kit autopsique préconisés par la SFTA (30), soit celui-ci était rajouté, dans un délai plus ou moins long, après la réalisation du prélèvement. La concentration de fluorure de sodium a ainsi varié selon les laboratoires entre 0,1 et $2 \%$. Pour mieux cerner ce problème, il serait nécessaire de savoir exactement à partir de quelle concentration le fluorure de sodium est réellement efficace.

Le $3^{\text {ime }}$ biais ouvre un débat très large, et concerne la validation pour application en toxicologie post-mortem des techniques mises en ouvre dans chaque laboratoire. Nous avons en effet mis en évidence, avec cette étude, que 5 molécules présentaient une augmentation apparente de leur concentration et que par ailleurs des molécules d'une même famille chimique présentaient des stabilités très différentes. L'augmentation apparente ne peut pas s'expliquer par un processus de réduction in vitro de métabolites oxydés comme cela est décrit parfois pour des cas réels comme la clozapine (16) puisque les échantillons dans le cas présent n'étaient pas surchargés en métabolites.

D'autres hypothèses peuvent être émises. Il peut s'agir d'un phénomène de fixation plus ou moins rapide de la molécule par adsorption sur la surface du contenant ou encore par liaison aux substances endogènes présentes (protéines, amines de putréfaction, ...) suivi d'une phase de défixation. La succession de ces processus soumis également aux différentes conditions de traitement des échantillons (temps d'homogénéisation lors des surcharges, sonication, extraction ...) pourrait être la cause de nombreuses disparités. Les échantillons post-mortem ayant des propriétés physico-chimiques particulières, il est primordial de prendre en compte l'effet matrice au moment du dosage, et une démarche de validation adaptée au domaine de la toxicologie post-mortem doit être proposée. Lorsque l'effet matrice est trop important, une quantification par ajouts dosés sur l'échantillon à doser pourrait être une solution (4). 


\section{Conclusion}

Les études sur échantillons surchargés sont complémentaires de celles réalisées à partir de cas réels. Les biais difficilement maîtrisables de cette étude ne sont que le reflet de notre pratique quotidienne. Nous sommes contraints de traiter régulièrement des prélèvements putréfiés et nous réceptionnons malheureusement encore des prélèvements sanguins réalisés sans adjonction de conservateur.

Enfin, ces travaux doivent nous inciter à poursuivre des études de stabilité à $+4^{\circ} \mathrm{C}$ ou $-20^{\circ} \mathrm{C}$ à partir de cas réels ; à œuvrer pour obtenir des prélèvements de la meilleure qualité possible avec un ajout systématique d'adjuvant de conservation (à concentration efficace) dès le moment des prélèvements d'autopsie et de conserver si possible les échantillons à $-20^{\circ} \mathrm{C}$, dans les phases pré et post analytiques.

\section{Références}

1. Pelissier-Alicot A.L., Gaulier J.M., Champsaur P., Marquet P. Mécanismes de la redistribution post-mortem des xénobiotiques : le point sur l'état actuel des connaissances. Ann. Toxicol. Anal. $2001 ; 13(1)$ : 1-17.

2. Jones G. Postmortem Toxicology. In : Moffat A.C., Osselton M.D. Widdop B. Clarke's analysis of drugs and poisons in pharmaceuticals, body fluids and postmortem material. Third edition, Phamaceutical Press. 2004 ; 94-108.

3. Drummer O.H., Gerostamoulos J. Postmortem drug analysis : analytical and toxicological aspects. Ther. Drug Monit. $2002 ; 24: 199-209$.

4. Skopp G. Preanalytic aspects in postmortem toxicology. Forensic Sci. Int. $2004 ; 142$ : 75-100.

5. Toennes S.W., Kauert G.F. Importance of vacutainer selection in forensic toxicological analysis of drugs of abuse. $\mathrm{J}$. Anal. Toxicol. 2001 ; 25 : 339-343.

6. Clauwaert K.M., Van Bocxlaer J.F., De Leenheer A.P. Stability study of the designers drugs "MDA, MDMA, and

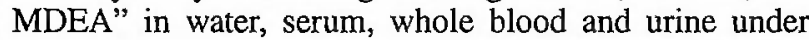
various storage temperatures. Forensic Sci. Int. $2001 ; 124$ : 36-42.

7. Al-Hadidi K.A., Oliver J.S. Stability of morphine and buprenorphine in whole blood. Int. J. Legal Med. 1998 ; $111: 165-7$.

8. Carroll F.T., Marraccini J.V., Lewis S., Wright W. Morphine-3-D glucuronide stability in postmortem specimens exposed to bacterial enzymatic hydrolysis. Am. J. Forensic Med. Pathol. 2000 ; 21 : 323-9.

9. Skopp G., Potsch L., Klingmann A., Mattern R. Stability of morphine,morphine-3-glucuronide and morphine-6glucuronide in fresh blood and plasma and post mortem blood samples. J. Anal. Toxicol. $2001 ; 25$ : 2-7.

10. Isenschmid D.S., Levine B.S., Caplan Y.H. A comprehensive study of the stability of cocaine and its metabolites. J. Anal. Toxicol. $1989 ; 13: 250-6$.

11.Pépin G., Dubourvieux N., Gaillard Y. Difficulté d'interprétation des taux des benzodiazépines et molécules appa- rentées dans le sang de cadavre prélevé à l'autopsie : étude in vitro après conservation pendant 6 mois à différentes températures. J Med Leg Droit Med. 1998 ; 41, 5 : 341-53.

12. Levine B., Blanke R.V., Valentour J.C. Postmortem stability of benzodiazepines in blood and tissues. J. Forensic Sci. $1983 ; 28: 102-15$.

13. Robertson D., Drummer OH. Stability of nitrobenzodiazepines in postmortem blood. J. Forensic Sci. 1998 ; 43 : 5-8.

14. Binsumait I.A., Hadidi K.A, Raghid S.A. Stability of fluoxetine in stored plasma, aqueous, and methanolic solutions determined by HPLC with UV detection. Pharmazie. $2001 ; 56(4), 311-313$.

15. Elliott S.P., Hale K.A. A previously unidentified acepromazine metabolite in humans : implications for the measurement of acepromazine in blood. J. Anal. Toxicol. $1999 ; 23: 367-371$.

16. Patrick K.S., Markowitz J.S. Potential for overestimation of clozapine concentrations. J. Anal. Toxicol. 1997 ; 21 : 73-4.

17. Olesen O.V., Linnet K. Determination of olanzapine in serum by high-performance liquid chromatography using ultraviolet detection considering the easy oxidability of the compound and the presence of other psychotropic drugs. J. Chromatogr. 1998 ; 714 : 309-315.

18. Stevens H.M. The stability of some drugs and poisons in putrefying human liver tissues. J. Forensic Sci. Soc. $1984 ; 24: 577-89$.

19. Takayasu T., Holterman K., Ohshima T., Pounder D.J. Stability of tricyclic antidepessants in formalin solutions. J. Forensic Sci. 1998 ; 43 : 1213-19.

20. Holmgren P., Druid H., Holmgren A., Ahlner J. Stability of drugs in stored postmortem femoral blood and vitreous humor. J. Forensic Sci. 2004 ; 49 (4), 820.

21. Berthault F., Tracqui A., Kintz P., Mangin P. Intoxication au trichloréthylène et conservation des prélèvements. Toxicorama. $1995 ; 7 ; 27-33$.

22. Jourdil N., Pinteur B., Vincent F., Marka C., Bessard G. Simultaneous determination of trimipramine and desmethyl- and hydroxy-trimipramine in plasma and red blood cells by capillary gas chromatography with nitrogen-selective detection. J. Chromatogr. 1993 ; 613 : 59-65.

23. Kintz P. Difficultés analytiques et d'interprétation dans les expertises toxicologiques liées à la présence de GHB . J. Med. Leg. Droit Med. 2002 ; 45 (6) : 293-297.

24. Dictionnaire Vidal 2005 : www.vidal.fr

25. Danel V., Pulse C., Castot A. Phénothiazines. In : Bismuth C., ed. Toxicologie clinique. Paris : Médecine-Sciences Flammarion. 2000 ; 144-7.

26. Danel V., Baud F., Castot A. Butyrophénones et benzamides substituées et autres neuroleptiques. In : Bismuth C., ed. Toxicologie clinique. Paris : Médecine-Sciences Flammarion. 2000 ; 148-55.

27. Danel V., Leborgne C., Castot A. Antidépresseurs. In : Bismuth C., ed. Toxicologie clinique. Paris : MédecineSciences Flammarion. 2000 ; 148-55.

28. Robertson D., Drummer O.H. Postmortem drug metabolism by bacteria. J. Forensic Sci. 1995 ; 40 : 382-386.

29. Askeland R.A., Morrison S.M. Cyanide production by Pseudomonas fluorescens and Pseudomonas aeruginosa. Appl Environ Microbiol 1983 Jun ; 45 (6) : 1802-7.

30. Pépin G., Deveaux M., Goullé J.P., Kintz P., Marquet P. Les prélèvements d'autopsie nécessaires à la bonne exécution des expertises toxicologiques. Toxicorama. 1998 ; $10: 110-19$. 